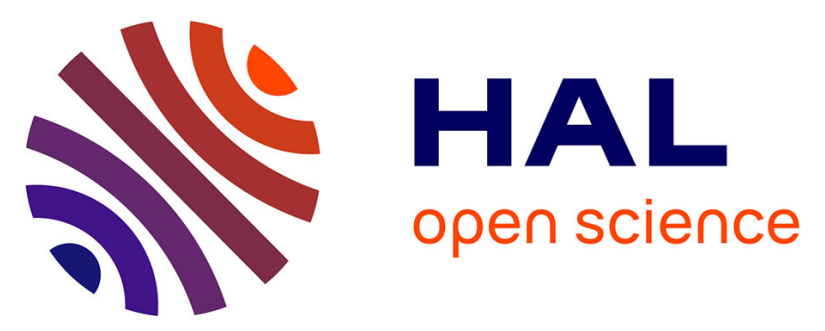

\title{
Matching filtering by region-based attributes on hierarchical structures for image co-segmentation
}

Francisco Javier Alvarez Padilla, Barbara Romaniuk, Benoît Naegel, Stéphanie Servagi-Vernat, Dimitri Papathanassiou, Nicolas Passat

\section{- To cite this version:}

Francisco Javier Alvarez Padilla, Barbara Romaniuk, Benoît Naegel, Stéphanie Servagi-Vernat, Dimitri Papathanassiou, et al.. Matching filtering by region-based attributes on hierarchical structures for image co-segmentation. International Conference on Image Processing (ICIP), 2018, Athens, Greece. pp.131-135, 10.1109/ICIP.2018.8451384 . hal-01706566

\section{HAL Id: hal-01706566 \\ https://hal.univ-reims.fr/hal-01706566}

Submitted on 12 Feb 2018

HAL is a multi-disciplinary open access archive for the deposit and dissemination of scientific research documents, whether they are published or not. The documents may come from teaching and research institutions in France or abroad, or from public or private research centers.
L'archive ouverte pluridisciplinaire HAL, est destinée au dépôt et à la diffusion de documents scientifiques de niveau recherche, publiés ou non, émanant des établissements d'enseignement et de recherche français ou étrangers, des laboratoires publics ou privés. 


\title{
MATCHING FILTERING BY REGION-BASED ATTRIBUTES ON HIERACHICAL STRUCTURES FOR IMAGE CO-SEGMENTATION
}

\author{
Francisco Javier Alvarez Padilla ${ }^{1}$, Barbara Romaniuk ${ }^{1}$, Benoît Naegel ${ }^{2}$, Stéphanie Servagi-Vernat ${ }^{1,3}$, \\ Dimitri Papathanassiou ${ }^{1,4}$ and Nicolas Passat ${ }^{1}$ \\ ${ }^{1}$ Université de Reims Champagne-Ardenne, CReSTIC, France \\ ${ }^{2}$ Université de Strasbourg, CNRS, ICube, France \\ ${ }^{3}$ Radiotherapy Department, Institut Jean-Godinot, Reims, France \\ ${ }^{4}$ Nuclear Medicine Department, Institut Jean-Godinot, Reims, France
}

\begin{abstract}
Inter / intra operator errors and high-time consumption induced by manual delineation, are the main drawbacks nowadays in clinical PET tumor segmentation. Several methodologies have been proposed to automate this task. However, there is not yet a validated general protocol to use in clinical routine. Multimodality imaging has been shown to provide good performance, taking into account both functional and anatomical scopes together for segmentation decision. In this context, the involved images used are generally required to be spatially corresponding. However, this is not always the case due to acquisition constraints or for multidate follow-up. In this work, we propose a spatially independent algorithm that avoids image pre-processing (e.g. image registration) or acquisition adjustments for multimodal segmentation. In particular, non-spatially correspondent images (such as multitemporal ones) can be directly exploited taking advantage of hierarchical image structure properties. Regions, obtained from hierarchical models of images, are coevaluated to match similar ones such as tumors on PET and CT. Results show good performance in terms of time-computing and robustnesses dealing with PET/CT segmentation problems such as necrosis, compared with other methodologies.
\end{abstract}

Index Terms - Matching, segmentation, multimodality, hierarchical models, region-based attributes.

\section{INTRODUCTION}

Positron Emission Tomography/Computed Tomography bimodal scope (PET/CT) is widely used to analyze body cellular metabolism for detection, diagnosis, treatment planning and follow-up of several diseases such as some kinds of cancers. The measurement of radiotracer uptake (mostly ${ }^{18} \mathrm{FDG}$ ) allows one to detect abnormal metabolic tissues and their aggressiveness level. Methodologically, CT coupling provides anatomic information used to correct PET attenuation, and an anatomical reference for functional/anatomical fused visualization. For radiotherapy treatment purposes, iodinebased contrast-enhanced CT (CE-CT) has a higher preference [1]. Contrast injection allows one to improve the contrast of tissues that are well vascularized, for instance tumors where blood transit is a factor that predisposes a higher contrast absorption and tumor cell

This work was funded by the University of Guadalajara, Mexico (expedient $021-V / 2015 / 660$ ) and by the French program Investissement d'Avenir run by the Agence Nationale pour la Recherche (Grant reference Infrastructure d'Avenir en Biologie Santé, ANR-11-INBS-0006). division. Some organs or anatomic structures surrounding the tumor can be vital and/or sensitive to radiation. Therefore, tumor zones are manually delineated by supervising anatomical information to apply the radiation protocol selected. However this modus operandi is relatively slow and can lead to intra-inter operator variations [2].

Several methodological solutions have been proposed [3] to automate and improve, in terms of robustness and ergonomy, tumor segmentation process, mainly on PET due to its high sensitivity and specificity. Thresholding approaches (fixed [4] and adaptive [5, 6]) are still mostly used in clinical routine, due to their straightforward implementation and the production of acceptable results. Other methodological fields have also presented their approaches with their respective limits and advantages: region growing [7], learningbased [8], gradient-based [9], watershed [10], active contours [11], Gaussian mixture models [12], FLAB [13], fuzzy-c-means [14], component-tree [15] and hybridization [16].

Recently, multimodality is gaining share. In $[17,18]$, it is claimed and experimentally confirmed that the use of semantically different and relevant information sources allows for a better segmentation than monomodal approaches. However, the majority of the methods relying on this paradigm require a spatial correspondence between images to be used. In particular, registration is required, which can be complex in certain cases, due to multimodality, multitime and/or different resolutions.

Based on these considerations, matching approaches can constitute an alternative methodology, although not much exploited yet for tumor recognition / segmentation issues [19]. These approaches have been mainly exploited for image retrieval [20], object recognition [21] and image registration [22] under the general objective of finding similar objects between images. Geometric-based features [23], General Fourier Descriptor and [24] Zernike moments [25] have been used as parameters to object characterization; and similarity assignment [26] has been defined taking into account the spatial (intrinsic features) and/or topography (inclusion and overlapped regions). By contrast, we propose to exploit this correlation paradigm from another point of view. Indeed, we aim to inter-relate similar regions from two (or more) images that are semantically different, in order to delete those which are not matched. This approach encompasses the use-case of different imaging types (mono- or multitemporal PET/CT), but also the case of synthetic images or binary masks that could be represented by pre-segmentation results, and associated to real target images, in a context of example-based segmentation. Moreover, hierarchical structures are exploited to define image regions, taking advantage of their intrinsic properties, such as spatial independence and spectral-spatial region partition. At the 
end of the matching process, the remaining nodes are evaluated by a thresholding protocol on PET (high tumor contrast) to identify the tumor nodes and retrieve the segmentation result.

\section{METHOD}

Our method uses the matching strategy as a way to obtain easier object segmentation, by discriminating information considered dissimilar on medical images.

Four main steps compose this methodology: tree (a.k.a hierarchical structure, HS) construction; attribute computing; match pruning; and image restitution (see Fig. 1).

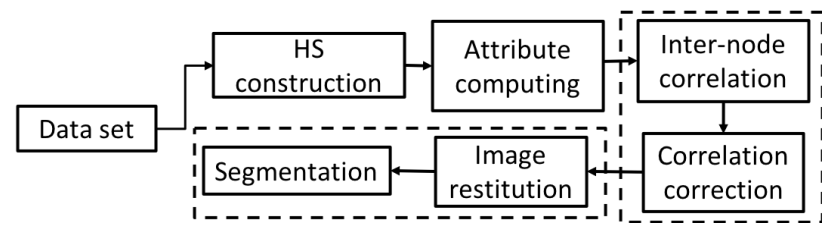

Fig. 1. Algorithm pipeline.

1. HS construction: From a set of $n \geq 2$ images, a hierarchical structure $\Psi$ is built for each image, leading to $n$ tree structures $\Psi_{1}, \Psi_{2}, \ldots, \Psi_{n}$. The nature of $\Psi$ depends of its relevance for modeling the objects of interest (OOI) (see Fig. 2). The image partition convention of $\Psi$ (e.g. max-tree, min-tree, tree of shapes, etc.) must guarantee that the OOI is defined inside the graph.

2. Attributes computing: Relevant attributes are computed [27] on the nodes of the tree $\Psi$ in order to characterize them. These features (generally expressed as scalar values) must be representative of the OOI in a global scope (indeed, intensity scales are not often directly comparable between imaging modalities).

3. Match pruning: The regions of the trees are co-related, with the purpose of finding the similar ones (see Fig. 2). Their similarity level is measured by a distance between node attributes, with a respective tolerance value $\varepsilon$. Regions which exhibit distance lower than a given $\varepsilon$ are then considered similar. Afterward, mismatchings are reduced by a correlation correction step, with the purpose of deleting false positives.

4. Segmentation protocol and image restitution: A protocol of segmentation is finally applied to identify the nodes belonging to the object of interest. These nodes are re-embedded in their initial image support space to recover the segmentation result.

\subsection{Hierarchical image structures}

A hierarchical structure (HS) is a graph representation of an image. This structure $\Psi$ is formed by nodes $k \in \Theta$ that are linked by edges $e \in E$. Formally, a node represents an image region which is formed by a set of $2 \mathrm{D}$ pixels / $3 \mathrm{D}$ voxels spatially connected and obtained according to a spectral partition. These nodes are hierarchically organized via an order relation, e.g. the inclusion relation, or the nested relation of their frontiers.

In this work, two hierarchical structures are mainly considered: the component-tree (C-T) $\tau$ and the tree of shapes $\Phi$. These hierarchical structures are chosen, in particular, with respect to their ability to model the mixed spatial/spectral properties of objects of interest in various kinds of medical imaging modalities.

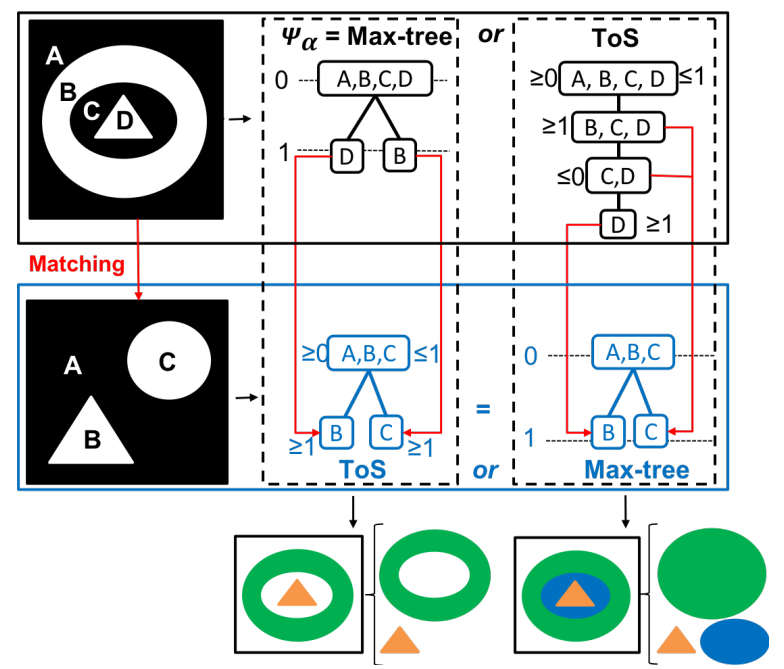

Fig. 2. An example of simple region matching by circularity attribute between a Max-tree (as $\Psi_{\alpha}$ ) and a Tree of Shapes, and vice versa, showing their results according to HS partition.

1. Component-tree : In this HS, images regions are obtained applying a sequence of thresholding ( $\geq$ order for a max-tree, $\leq$ for a min-tree) at each intensity level $\lambda_{*}(I)$, leading to image partition [28]. From the highest level $\lambda_{\max }(I)$ to the lowest level $\lambda_{\min }(I)$, nodes are related by spatial inclusion.

2. Tree of shapes : The $\operatorname{ToS} \Phi$ [29] is defined as a self-dual version of the $\mathrm{C}-\mathrm{T}$, that merges the max- and min-trees. The fusion of the two associated partitions, following a spatial inclusion order of hierarchy, provides a "no-hole" node structure which defines an image isocountour representation.

These two structures can be computed in quasi-linear time [30, 31].

\subsection{Attributes}

An attribute [32] is a feature used to characterize image regions. These features can rely on different notions, e.g. spectral attributes, spatial attributes, geometrical attributes or hybrid attributes (i.e. a mixture of the above). Attributes $h$ are stored, for each node / region of the trees, in a vector $A=\left[h_{1}, h_{2}, \ldots, h_{H}\right]$. They have to be inter-graph normalized according to their value scale.

\subsection{Match pruning}

\subsubsection{Inter-node-tree correlation}

This step aims to find the potentially correlated nodes. From the tree set $\Psi_{1: n}$, one of its tree is selected, and is called $\Psi_{\alpha}$, while the others are denoted $\Psi_{r e f=1: n-1}$. The nodes $\Theta_{\alpha}$ of $\Psi_{\alpha}$ are intercorrelated with the nodes $\Theta_{r e f *}$ of the remaining trees $\Psi_{r e f *}$. The comparison queue for each node $k_{i} \in \Theta_{\alpha}$ is comprised by the nodes $k_{j} \in \Theta_{\text {ref }}$ of trees $\Psi_{\text {ref }}$. An Euclidean distance $D\left(\overrightarrow{A_{i}}, \overrightarrow{A_{j}}\right)$ is computed between inter-node attributes $A$ :

$$
D\left(\overrightarrow{A_{i}}, \overrightarrow{A_{j}}\right)=\sum_{a=1}^{H} \beta[n]\left(A_{i}[a]-A_{j}[a]\right)
$$

where $\beta$ is a vector of size $m$ that weights up each attribute according to its reletance, with $\sum_{a=1}^{H} \beta[a]=1$. 
A node status $M$ is defined for nodes $\Theta_{\alpha}$ and a matching matrix $m_{i j}$ is created to store the binomial relation $i j$. In particular, if $\left.D\left(\vec{A}_{i}, \overrightarrow{A_{j}}\right)\right)<\varepsilon$, then we set $k_{i}(M)=$ true, achieving a similarity matching which is set at $m_{i j}$. At the end of the queue, if $k_{i}$ does not reach at least one $m_{i j}$, then we set $k_{i}(M)=$ inactive. The $\varepsilon$ is a user-defined parameter chosen within the interval $[0,1] \in \mathbb{R}^{+}$that establishes the similarity threshold.

\subsubsection{Correlation correction}

More than one node $k_{j}$ can be matched with a node $k_{i}$ if they accomplish the marked threshold. Two post-processing corrections are considered in order to refine the results obtained from the above approach, namely hierarchical privilege and best representative.

A matching crossed problem occurs when an ancestor node (higher $\lambda *$ ) $A n c_{i}$ of $k_{i}$ in $\Psi_{A}$ is related with $k_{j}$ in $\Psi_{B}$ and an ancestor $A n c_{j}$ of $k_{j}$ is related with $k_{i}$. Respecting their hierarchy, in an unilateral decision favoring $\alpha$, the $m_{i j}\left(k_{i}, A n c_{j}\right)$ is erased (see Fig. 3).
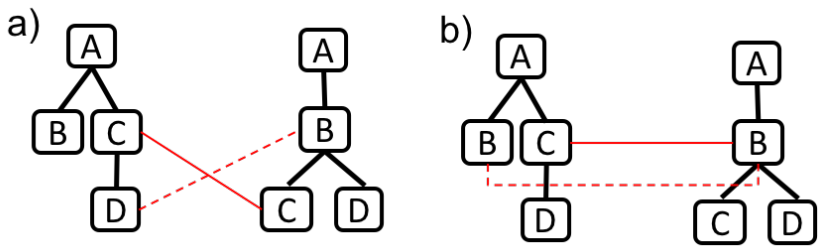

Fig. 3. a) Hierarchical priority and b) best representative corrections. Red lines are the correlation links $m_{i j}$ where the pointed ones are suppressed at the correction process.

The relation $m_{j i}$ is reevaluated comparing the remaining nodelinks to choose their best representative and to have a relation ratio 1:1. To this end, $k_{j}^{r e f}$ preserves the link $m_{j i}$ with $k_{i}$ which has the lowest $D\left(\overrightarrow{A_{i}}, \overrightarrow{A_{j}}\right)$. After these corrections, if a node $k_{i}$ loses all its inter-node relations $m_{j i}$ then $k_{i}(M)=$ inactive.

\subsection{Image restitution}

For the nodes such that $k_{i}(M)=$ active, a segmentation protocol is finally applied to select those ones that correspond to the OOI. The $\Psi_{\alpha}$ is reconstituted in its matrix image structure $I$, by pruning all nodes, except the selected ones. A direct image restitution is applied replacing node voxels at their orignal matricial place with an intensity value set at 1 and for the rest at 0 .

\section{INSTANTIATION FOR TUMOR MATCHING IN MEDICAL IMAGES}

The above methodology is adapted and implemented for tumor segmentation on medical PET/CT imaging.

\subsection{Hierarchical structure selection}

The tree of shapes is selected for both images, PET and CE-CT. This data-structure allows us to discriminate possible heterogeneities inside tumor regions.

In PET images, tumor cell biomarker metabolism is heterogeneous. In particular, if there is a necrosis (cell death), tumor composition has low intensities. In CT images, tumors can have a low contrast compared to neighboring tissues and if a contrast agent is injected, its absorption is irregular depending of vascularization.

These constraints can be fought by using isocontour notion when defining image partition. Thus, we can enssure that the OOI (tumor) is "well" represented inside the graphs with some regional variations due to acquisition mode specificities.

\subsection{Potential attributes}

Spectral attributes: It is not straighforward to compare images with different gray level scales, in particular SUV (PET) and Hounsfield units (CT) in our case. Nevertheless, the use of their derived units is possible such as Contrast $=\mid$ max_value - min_value $\mid$.

Spatial attributes : A normalization of space is required. The area value (number of voxels) is multiplied by the $3 \mathrm{D}$ image spacing $\left(\mathrm{mm}^{3}\right)$ for space arrangement.

For barycenter coordinates and other potential attributes derived from 3D spatial coordinates, image framework is normalized by creating a bounding box of human body having the brain and the bladder as top and bottom limits, respectively. A distance between barycenter coordinates (BCD) can be computed as a parameter to evaluate the relative dispersion of nodes.

Geometrical attributes: This is the most relevant group of attributes due to its spatial-spectral independence. Shape features can be easily evaluated according to voxels distribution at the node.

- Compactness $=4 \pi *$ area / contour length ${ }^{2}$, is the circularity (2D) / sphericity (3D) level, an often used feature in tumors.

- Complexity = contour length / area.

- Rectangularity = area/bb_area, where bb_area is the bounding box area which is defined by the supremum and the infimum points.

\subsection{Segmentation protocol}

PET intensities are used to identify the object of interest in the tree. A threshold $t$ of $S U V_{\max }$ is applied on PET image. Voxels that have an intensity value higher than $t$ are mapped with their correspondent $T$ nodes $k^{t}$ in $\operatorname{ToS}_{P E T}$. If $\operatorname{ToS}_{P E T}=\Psi_{\text {ref }}$, following the link $m_{j i}$, the $k_{j}^{t}$ are projected to $\Psi_{\alpha}$ to identify their respective $k_{i}^{t}$. If this node $k_{i}^{t}(M)=$ inactive, the node mapping is redirected, way down into the root, assigning the next active node. The $k_{1}^{t} \cup k_{2}^{t} \ldots \cup k_{T}^{t}$ is selected to represent the segmentation.

Actually, this threshold protocol has a semantically different meaning compared with a normal threshold on image intensities. Voxels that respect the threshold protocol can be placed in a node where other voxels do not.

\section{EXPERIMENTATION}

\subsection{Data}

Our material of experimentation is composed of ${ }^{18} F$-FDG PET-CT with iodinated contrast enhancement (CE) of different cancer patients (neck and head cancer, colon cancer). Images were carried out by using a GE Discovery 710 PET/CT scanner at the Cancerology Institute Jean-Godinot in Reims, France.

Patients received an intravenous dose of FDG (3 MBq/kg). Then, 50 minutes after, CT phase was performed first, 70 seconds after an intravenous dose of iodinated contrast agent (Optiject 350), with auto $\mathrm{mA}$ mode, adaptive statistical iterative reconstruction 

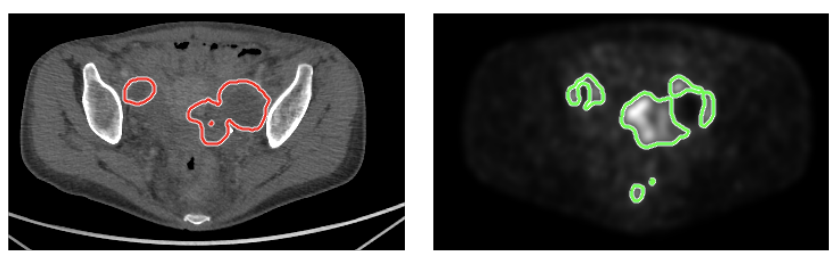

Fig. 4. CE-CT on the left and PET on the right. In red, a manual delineation provided by a radiotherapist; in green, a threshold protocol of $S U V_{\max }$, the most common methods nowadays in clinical routine.

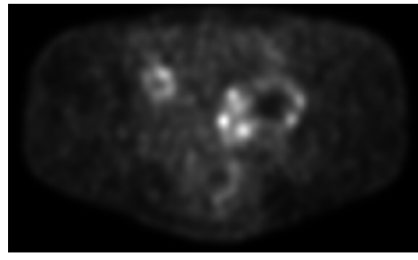

a)

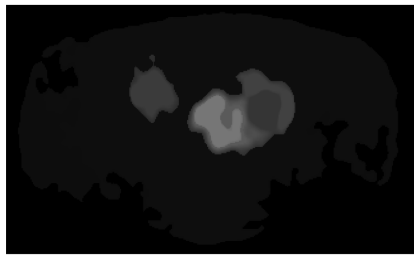

c)

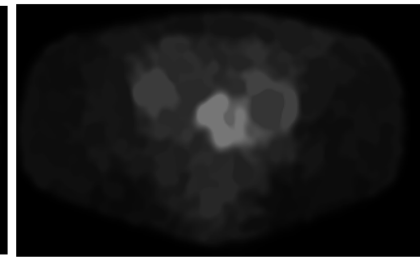

b)

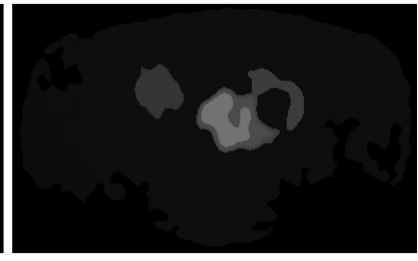

d)
Fig. 5. Regions of ToS partition of a) at $\Psi_{\alpha}^{P E T}, \Psi_{r e f}^{C E-C T}$ that are matched according to $\mathrm{a} \varepsilon=\mathrm{b}) 0.1$, c) 0.05 and d) 0.01 .

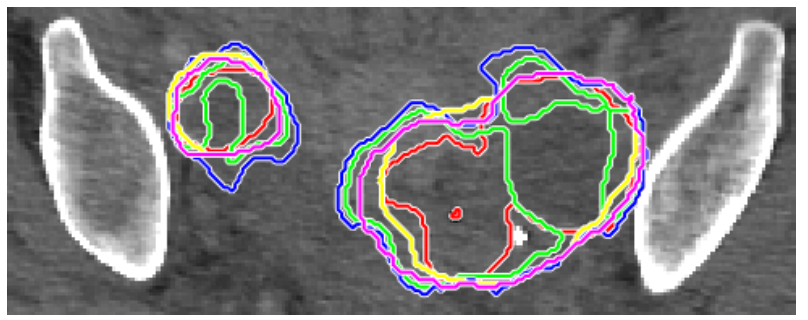

Fig. 6. On blue $\Phi_{\alpha}^{P E T}, \Phi_{\text {ref }}^{C E-C T}$, on yellow $\Phi_{\alpha}^{C E_{C} T}, \Phi_{\text {ref }}^{P E T}$, and magenta $\Phi_{\alpha}^{P E T}, \Phi_{\text {ref }}^{B M}$, results obtained.

(ASIR) and native collimation of $16 \times 1.25 \mathrm{~mm}$. PET phase was sequentially performed and reconstructed using an iterative algorithm (OSEM 24 subsets, 2 iterations) and post-filtered with a Butterworth filter (cut-off frequency: $6.4 \mathrm{~mm}$ ). The resulting matrices spaces are $256 \times 256 \times 263$ with voxel spacing of $2.73 \times 2.73 \times 3.27 \mathrm{~mm}^{3}$ for $\mathrm{CT}$ and $512 \times 512 \times 343$ with a voxel spacing of $0.97 \times 0.97 \times 2.5$ $\mathrm{mm}^{3}$ for PET.

\subsection{Implementation}

A ToS $\Phi$ is built from each PET and CE-CT. We experiment both cases using $\Phi_{\alpha}^{P E T}, \Phi_{r e f}^{C E-C T}$ and vice versa. A third combination is tested, $\Phi_{\alpha}^{P E T}$ is built and a binary mask obtained by operator delineation was used as $\Phi_{\text {ref }}^{B M}$. The attributes explained in Sec. 3.2 (with exception of spectral ones and rectangularity) are computed, $A=[$ compacity, area, complexity, $B C D]$. The weighting vector $\beta$ was set at $[0.25,0.2,0.25,0.3]$ giving a slightly higher importance to the distance between region centroids than size.

The $\varepsilon$ is interactively moved, thus analyzing in real time, several possible results (see Fig. 5). The lower $\varepsilon$, the lower the dissimilarity tolerance, and the number of nodes matched.

\subsection{Computer performances}

For these experiments, a computer with a processor 3,1 GHz, Intel Core i7 and Memory 16 Go $2133 \mathrm{MHz}$ was used. The matching step takes 6.36 seconds, in avereage. The whole process, including 2 ToS construction for medical images (resolution before mentioned), 4 attributes computing and image restitution takes 21.70 seconds, in average.

\subsection{Results}

Our results were compared with other methodologies of the state of the art, including the computation of the $40 \% S U V_{\max }$ and two versions of multi-modal Random Walker [33], scalar RW-PET/CT (intensities values) and vectorial HFA RW-PET/CT (Hierarchical Forest Attributes - vectorial values)

Table 1. Methodological comparison

\begin{tabular}{|l|c|l|c|}
\hline & DSC $(\mu \pm \sigma)$ & Our method $(\alpha$, ref $)$ & DSC $(\mu \pm \sigma)$ \\
\hline $40 \% S U V_{\max }$ & $0.551 \pm 0.179$ & PET, CT & $0.744 \pm 0.052$ \\
\hline RW-PET/CT & $0.802 \pm 0.038$ & CT, PET & $0.781 \pm 0.040$ \\
\hline HFA RW-PET/CT & $0.856 \pm 0.036$ & PET, BM & $0.773 \pm 0.045$ \\
\hline
\end{tabular}

\section{DISCUSSION}

This treatment is easy and fast. Results show a greater spatial accuracy than threshold methods, but a lower one than other more complex methods. However, the computation time is much lower, compared to these other methods. Based on this trade-off between time consumption and result quality, the proposed method seems to provide a convenient solution to compare two images in real-time and could be applied easily in clinical routine.

Spatial correspondence handling between medical images is generally a complex issue in multimodal segmentation; and their registration is time-consuming and sometimes prone to errors. Tree of shapes computing is fast and a propitious solution for nonspatially correspondent object evaluation. This structure is spatially independent once built. In other words, objects of interest can be equally distributed in the graph; no matter the image spatial resolution or local displacements according to a background.

We presented a fully automated methodology. The expert intervention is only required to define few parameters according to the object-of-interest characteristics. Using the same parameters, the result is reproducible, which avoids inter/intra operator errors. The particularities of this similarity matching methodology lead us to confirm that hyperfixant and transit organs are segmented too, since they have the same conditions as the OOI (tumors) on PET/CE-CT, a functional-anatomic representation; for the use of preceding segmentation masks $(B M)$, this problem does not happen. This analysis of matching is only spatial and not topological, because interimage structures are not assured to have the same internal consistence. Results lead us to continue this track crossing to increase robustness in tumor matching on medical images. 


\section{REFERENCES}

[1] Antoch G., Freudenberg L., Beyer T, Bockisch A. and Debatin J. To Enhance or Not to Enhance? $18 F-F D G$ and CT Contrast Agents in Dual-Modality 18F-FDG PET/CT. J Nucl Med, 45:5665, 2004.

[2] Fox J., Rengan R., OMeara W., Yorke E., Erdi Y., Nehmeh S., Leibel S. and Rosenzweig K. Does registration of pet and planning ct images decrease interobserver and intraobserver variation in delineating tumor volumes for non-small-cell lung cancer? Int J Radiat Oncol, 62:70-75, 2005.

[3] Foster B., Bagci U., Mansoor A., Xu Z. and Mollura D. A review on segmentation of positron emission tomography images. Computers in Biology and Medicine, 50:76-96, 2014.

[4] Yu W., Fu X.-L., Zhang Y. J., Xiang J.-Q., Shen L., Jiang G.-L. and Chang J. GTV spatial conformity between different delineation methods by 18 FDG PET/CT and pathology in esophageal cancer. Radiother Oncol, 93(3):441-446, 2009.

[5] Drever L., Roa W., McEwan A. and Robinson D. Iterative threshold segmentation for pet target volume delineation. Med Phys, 34:1253-1265, 2007.

[6] Nestle U., Schaefer-Schuler A., Kremp S., Groeschel A., Hellwig D., Rübe C. and Kirsch C.-M. Target volume definition for $18 F-F D G$ PET-positive lymph nodes in radiotherapy of patients with non-small cell lung cancer. Eur J Nucl Med Mol I, 34:453462, 2007.

[7] Day E., Betler J., Parda D., Reitz B., Kirichenko A., Mohammadi S. and Miften M. A region growing method for tumor volume segmentation on pet images for rectal and anal cancer patients. Med Phys, 36:4349-4358, 2009.

[8] Bi L., Kim J., Feng D. and Fulham M. Multi-stage thresholded region classification for whole-body PET-CT lymphoma studies. MICCAI, 569-576, 2014.

[9] Wanet M., Lee A., Weynand B., De Bast M., Poncelet A., Lacroix V., Coche E., Grégoire V. and Geets X. Gradient-based delineation of the primary GTV on FDG-PET in non-small cell lung cancer: A comparison with threshold-based approaches, $C T$ and surgical specimens. Radiother Oncol, 98:117-125, 2011.

[10] Tylski P., Bonniaud G., Decencière E., Stawiaski J., Coulot J., Lefkopoulos D. and Ricard M. ${ }^{18}$ F-FDG PET images segmentation using morphological watershed: A phantom study. NSSCR, 4:2063-2067, 2006.

[11] Li H., Thorstad W., Biehl K., Laforest R., Su Y., Shoghi K., Donnelly E., Low D. and Lu W. A novel pet tumor delineation method based on adaptive region-growing and dual-front active contours. Med Phys, 35:3711-3721, 2008.

[12] Aristophanous M., Penney B., Martel M. and Pelizzari C. A gaussian mixture model for definition of lung tumor volumes in positron emission tomography. Med Phys. 34:42234235, 2007.

[13] Hatt M., Cheze le Rest C., Descourt P., Dekker A., De Ruysscher D., Oellers M., Lambin P., Pradier O. and Visvikis D. Accurate automatic delineation of heterogeneous functional volumes in positron emission tomography for oncology applications. Int $\mathrm{J}$ Radiat Oncol, 77:301-308, 2010.

[14] Belhassen S. and Zaidi H. A novel fuzzy C-means algorithm for unsupervised heterogeneous tumor quantification in PET. Med Phys, 37:1309-1324, 2010.
[15] Grossiord E., Talbot H., Passat N., Meignan M. and Najman L. Automated $3 D$ lymphoma lesion segmentation from PET/CT characteristics. ISBI, 174-178, 2107.

[16] Dewalle-Vignion A.-S., Betrouni N., Lopes R., Huglo D., Stute $\mathrm{S}$. and Vermandel M. A new method for volume segmentation of PET images, based on possibility theory. IEEE T Med Imaging, 30:409-423, 2011.

[17] Bagci U., Udupa J., Mendhiratta N., Foster B., Xu Z., Yao J., Chen X. and Mollura D. Joint segmentation of anatomical and functional images: Applications in quantification of lesions from PET, PET-CT, MRI-PET, and MRI-PET-CT images. Med Image Anal, 17:929-945, 2013.

[18] Ju W., Xiang D., Zhang B., Wang L., Kopriva I. and Chen X. Random walk and graph cut for co-segmentation of lung tumor on PET-CT images. IEEE T Image Process, 24:5854-5867, 2015.

[19] Petrakis E. Design and evaluation of spatial similarity approaches for image retrieval. Image Vision Comput, 20:59-76, 2002.

[20] El-Kwae E. and Kabuka M. A robust framework for contentbased retrieval by spatial similarity in image data-bases. ACM Trans Information Systems, 17:174-198, 1999.

[21] Belongie S., Malik J. and Puzicha J. Shape matching and object recognition using shape contexts. IEEE T Pattern Anal, 24:509$522,2002$.

[22] Shen D. Image registration by local histogram matching. Pattern Recogn, 40:1161-1172, 2007.

[23] Alajlan N. and Kamel M. Geometry-based Image retrieval in binary image databases. IEEE T Pattern Anal, 30:1003-1013, 2008.

[24] Zhang D. and Lu G. Shape-based image retrieval using generic Fourier descriptor. Signal Processing: Image Communication, 17:825-848, 2002.

[25] Whoi-Yul K. and Yong-Sung K. A region-based shape descriptor using Zernike moments. Signal Processing: Image Communication, 16:95-102, 2000.

[26] Mattes J., Richard M. and Demongeot J. Tree representation for image matching and object recognition. DGCI, 298-309, 1999.

[27] Alvarez F., Grossiord É., Romaniuk B., Naegel B., Kurtz C., Talbot H., Najman L., Guillemot R., Papathanassiou D. and Passat N. Multicriteria 3D PET image segmentation. IPTA, 346-351, 2015.

[28] Salembier P., Oliveras A. and Garrido L. Anti-extensive connected operators for image and sequence processing. IEEE Transactions on Image Processing, 7:555-570, 1998.

[29] Ballester C., Caselles V. and Monasse P. The tree of shapes of an image. ESAIM, 9:1-18, 2003.

[30] Carlinet E. and Géraud T. A comparison of many max-tree computation algorithms. ISMM, 73-85, 2013.

[31] Géraud T., Carlinet E., Crozet S. and Najman L. A quasi-linear algorithm to compute the tree of shapes of $n-D$ images. ISMM, 97-108, 2013.

[32] Breen E. and Jones R. An attribute-based approach to mathematical morphology. Comp Imag Vis, 5:41-48, 1996.

[33] Alvarez F., Romaniuk B., Naegel B., Servagi-Vernat S., Morland D., Papathanassiou D. and Passat N. Hierarchical forest attributes for multimodal tumor segmentation on FDGPET/contrast-enhanced CT ISBI, Washington, USA, 2018. 